\title{
¿Qué problemáticas sociales y patrimoniales hay en tu entorno? Propuesta de trabajo integrado para los Grados de Educación Infantil y Primaria
}

\section{What social and patrimonial problems are there in your environment? Proposal of integrated work for Pre-Service Childhood and Primary Teachers'}

ELISA NAVARRO-MEDINA

ORCID: http://orcid.org/0000-0001-5523-7097

Universidad de Sevilla, Departamento

de Didáctica de las Ciencias

Experimentales y sociales.

enavarro5@us.es

Fecha de recepción: 09-11-2019.

Fecha de aceptación: 15-11-2019.

DOI: http://dx.doi.org/10.12795/9788447221912.064

Pp.: 1474-1499 
El trabajo que se presenta recoge el diseño de un Ciclo de Mejora Integrado para dos asignaturas pertenecientes a los Grados de Educación Infantil y Primaria. La propuesta experimentada, vinculada a la investigación de problemáticas sociales y patrimoniales, la secuencia de actividades realizada y la evolución de las ideas de los estudiantes, han puesto de manifiesto que se ha logrado una mejora significativa en el aprendizaje de los estudiantes y en el desarrollo profesional de las docentes participantes, consolidando formas de trabajo poco habituales en el contexto universitario, basadas en la coordinación y la evaluación entre pares.

Palabras clave: Didáctica de las Ciencias Sociales, Grado en Educación Primaria, docencia universitaria, experimentación docente universitaria, problemas sociales

\section{Abstract}

The work presented includes the design of a improvement cycle integrated into two subjects belonging to the Grades of Infant and Primary Education. The work problem, linked to the identification of social and patrimonial problems, the sequence of activities carried out and the evolution of the students' ideas has shown that these proposals represent an improvement for the students' learning and for the professional development of the participating teachers and consolidate a form of work that is unusual in the university context, based on coordination and peer evaluation.

Keywords: Social Science Didactics, Grade in Primary Education, university teaching, university teaching experimentation, social problems..

Jornadas de Formación e Innovación Docente del Profesorado | № 2 (2019) Esta obra se distribuye con la licencia Creative Commons 


\section{Descripción del contexto}

Las asignaturas en la que se ha implementado el CIMA integrado han sido Didáctica de las Ciencias Sociales, del tercer curso del Grado de Educación Primaria y Didáctica del Patrimonio Cultural de Andalucía de 40 curso en el Grado de Educación Infantil. En este capítulo presentaremos la repercusiones que ha tenido en la primera de ellas. Las clases se han organizado en dos sesiones de hora y veinte con el grupo completo y una sesión de hora y veinte con cada mitad del grupo. Han asistido el total de estudiantes matriculados $(n=72)$. La materia ha estado organizada en cinco problemas de trabajo que recogen las cuestiones básicas del perfil profesional del maestro/a del área de Educación Primaria. En el desarrollo de las sesiones (donde no se distingue entre clases teóricas ni prácticas) se ha ido conjugando la reflexión sobre los problemas prácticos profesionales seleccionados, con el diseño, aplicación y análisis de propuestas didácticas concretas para un aula de Primaria. La síntesis de la asignatura ha quedado recogida en la elaboración en pequeño grupo de un material curricular para el área de Ciencias Sociales en Primaria.

Esta asignatura la llevo trabajando desde hace 7 años y creo que he conseguido definir un modelo metodológico estable (aunando mi modelo posible e ideal) que ya se ha recogido en CIMA anteriores (Navarro-Medina, 2017; Navarro-Medina, 2018), por lo que los cambios realizados son pequeñas adaptaciones en lo relativo a algunas actividades de contraste que permitan superar a los estudiantes los obstáculos detectados en evaluaciones anteriores.

Sin embargo, el cambio fundamental de este año se ha basado en la realización de un CIMA integrado para el primer problema de la asignatura y que, junto con la asignatura de Infantil, ha roto con la dinámica de trabajo aislado al que está acostumbrado el docente universitario (Gómez, 
Escofet y Freixa, 2014). La justificación de esta propuesta se debe a varios motivos:

Los estudiantes viven ajenos a las realidades que no son las suyas.

El entorno social es complejo y su fragmentación en asignaturas provoca una desconexión entre los contenidos de la universidad -académicos-, los contenidos de la escuela -escolares-, y la realidad social.

Desde la universidad es necesario abordar la complejidad de la realidad escolar, a través de prácticas que pongan en juego en desarrollo de habilidades y competencias diversas.

Las propuestas de contenido presentadas a los estudiantes, y futuros maestros/as, deben hacerles ver que, aun estando en una formación inicial, requiere de ellos pensar en su desarrollo profesional como docentes.

Es preciso hacer converger a estudiantes de distintos Grados y asignaturas, con la intención de trabajar de forma conjunta los problemas del entorno, de la escuela y de la formación docente.

\section{Mapa de contenidos y problemas}

La integración de las dos asignaturas que se ha realizado parte, como se ha dicho, de la necesidad que tienen nuestros estudiantes de comprender que los problemas sociales y patrimoniales son complementarios y están presentes en su entorno más próximo. El traspaso de estas problemáticas a un aula pasa porque los futuros maestros y maestras conozcan, analicen, valoren y apuesten por un modelo didáctico que huya de planteamientos tradicionales y se enmarque en una perspectiva investigativa. Para 
ello, hemos incluido como contenidos de trabajo fundamental el Modelo Didáctico de Investigación (García, 2000) que promueve, por un lado, la investigación del docente (o futuro docente en nuestro caso) y la construcción de un modelo didáctico personal en evolución; y por otro, el progreso de los estudiantes (ahora estudiantes del Grado, más adelante maestros de niños y niñas) alejado de planteamientos de reproducción social y apostando por un modelo de ciudadanía activa y participativa (Bolivar, 2016). En resumen, abordamos todo esto desde una perspectiva de planificación, diseño y aplicación del modelo investigativo al día a día del aula (véase figura 1).

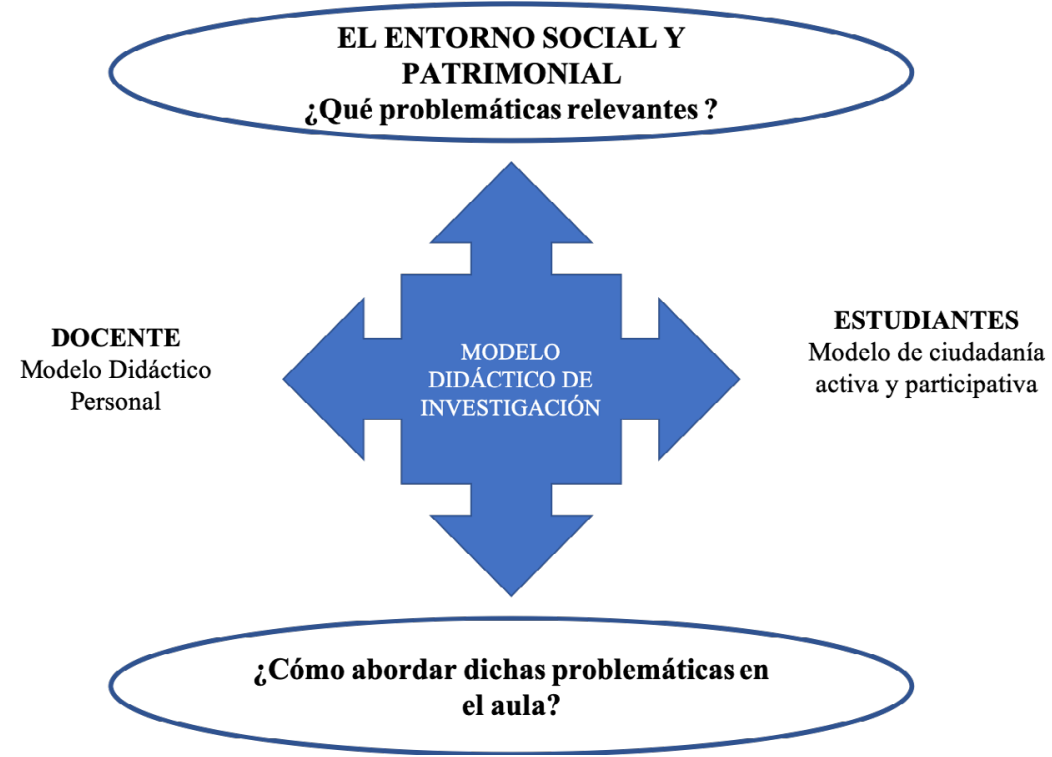

Figura 1. Planteamiento teórico del CIMA integrado

Este mapa más general se ha desarrollado en un mapa de contenidos más específico, donde podemos encontrar las preguntas-problema que guían el trabajo de los estudiantes, con los contenidos conceptuales (en forma de óvalos), los contenidos procedimentales (en forma de triángulo) y los contenidos actitudinales (en forma de hexágono). 
ELISA NAVARRO-MEDINA
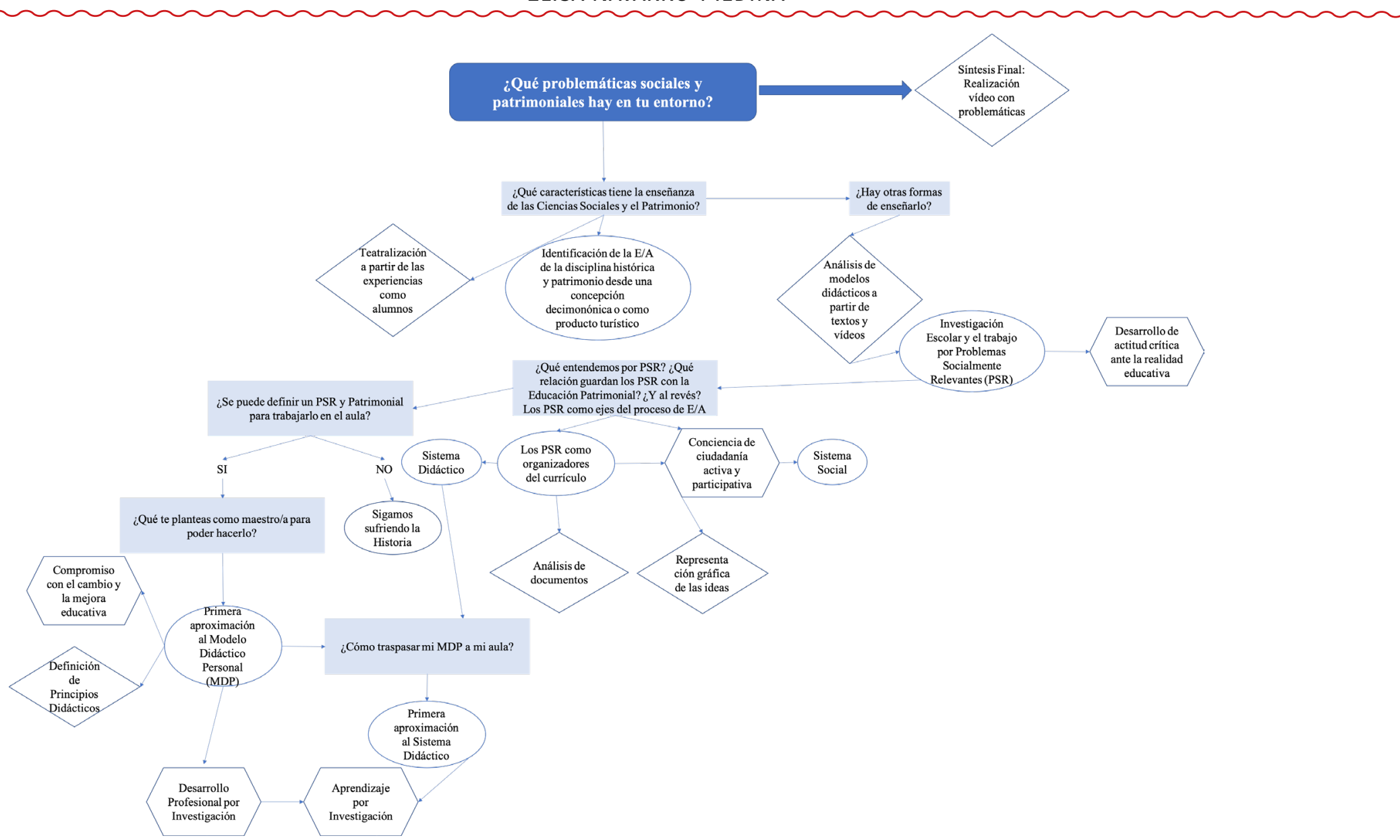

Figura 2. Mapa de contenidos del problema: ¿Qué problemáticas sociales y patrimoniales hay en tu entorno?

Jornadas de Formación e Innovación Docente del Profesorado | № 2 (2019)

Internacional (CC BY-NC-ND 4.0.) 


\section{ELISA NAVARRO-MEDINA}

De este modo, y al modificar el primer problema, el mapa general de la asignatura ha quedado del siguiente modo:

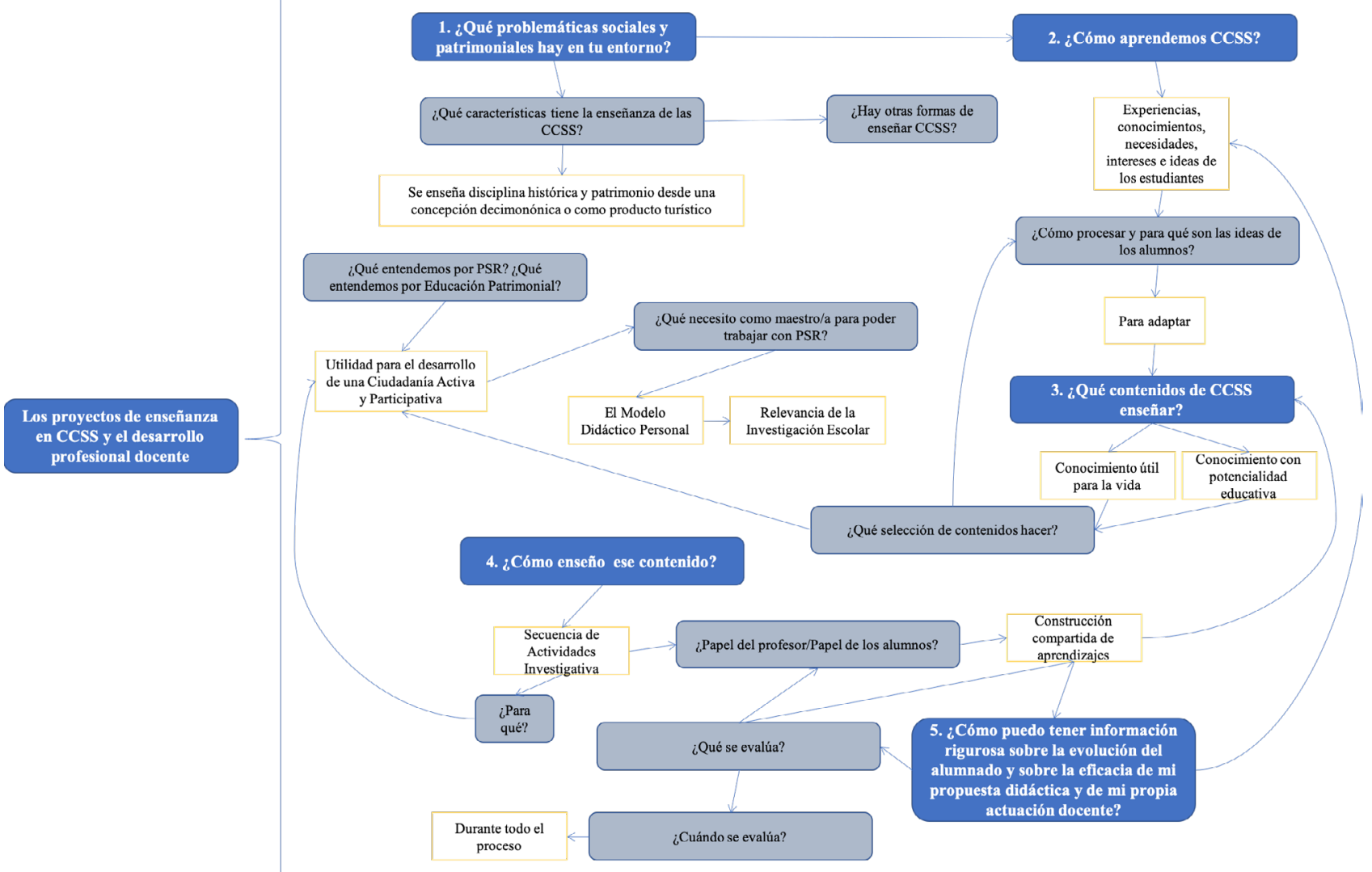

Figura 3. Mapa de contenidos general de la asignatura de Didáctica de las Ciencias Sociales para el Grado en Educación Primaria

Jornadas de Formación e Innovación Docente del Profesorado | № 2 (2019) 


\section{Modelo metodológico posible y secuencia de actividades}

Para el desarrollo de este CIMA integrado y teniendo en cuenta el bagaje que ambas docentes teníamos, establecimos un modelo metodológico común que ha partido del establecimiento de un problema, seguido de la obtención y análisis de las ideas de los estudiantes. El problema general se ha subdividido en distintos subproblemas, más específicos, para los que se han desarrollado secuencias de actividades investigativas que han permitido abordarlo. De las conclusiones parciales obtenidas de los subproblemas, se ha vuelto a interrogar a los estudiantes por sus ideas, concluyendo en una resolución del problema general.

Jornadas de Formación e Innovación Docente del Profesorado | № 2 (2019) Esta obra se distribuye con la licencia Creative Commons Reconocimiento-NoComercial-SinObraDerivada 4.0 Internacional (CC BY-NC-ND 4.0.) 
ELISA NAVARRO-MEDINA

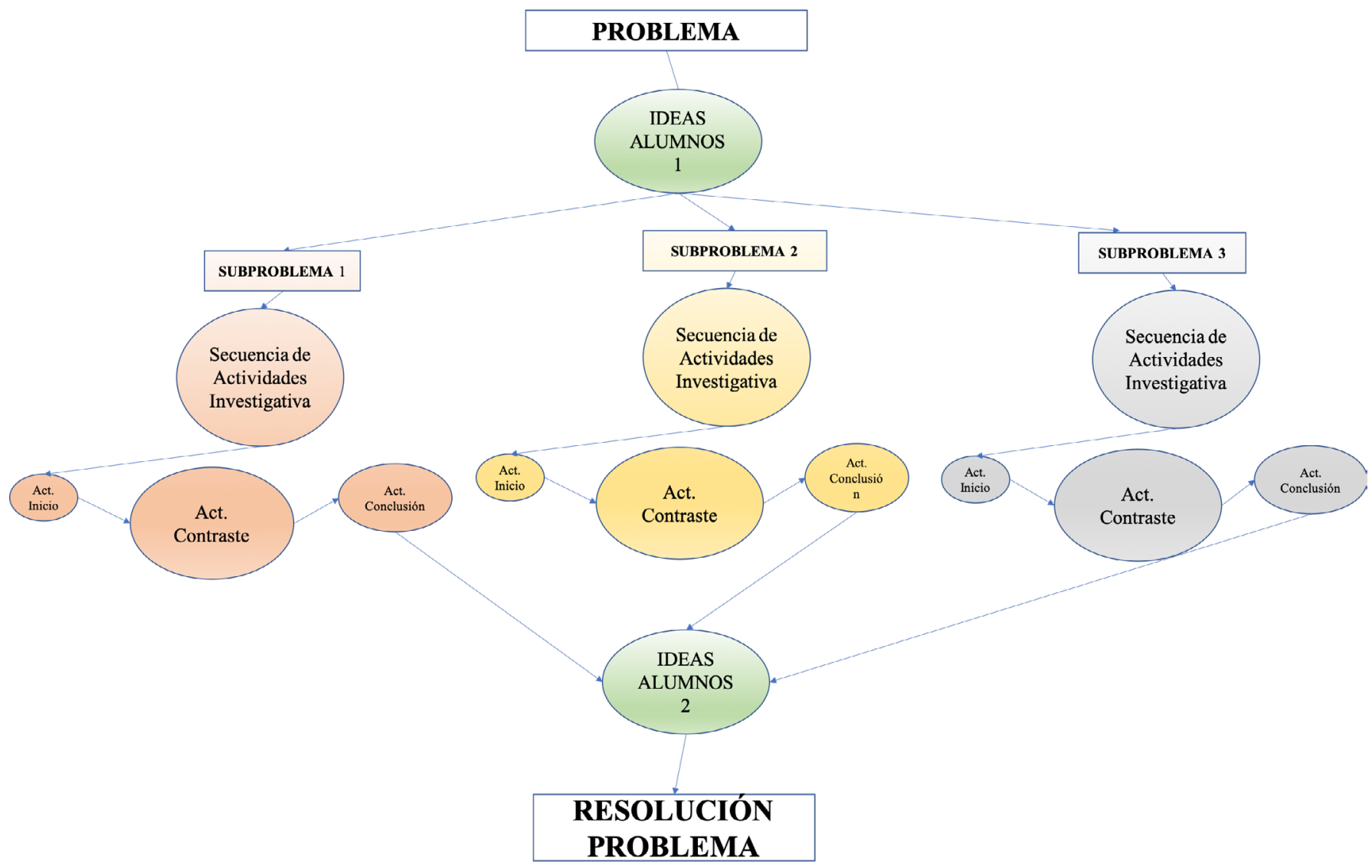

Figura 3. Mapa de contenidos general de la asignatura de Didáctica de las Ciencias Sociales para el Grado en Educación Primaria

Jornadas de Formación e Innovación Docente del Profesorado I № 2 (2019) 
El modelo metodológico anterior se ha concretado en la secuencia de actividades que se recoge en la tabla 1. Tabla 1. Secuencia de actividades para el problema ¿Qué problemáticas sociales y patrimoniales hay en tu entorno?

\begin{tabular}{|c|c|c|}
\hline \multicolumn{3}{|l|}{ SESIÓN 1. PRESENTACIÓN } \\
\hline TIPO DE ACTIVIDAD & EXPLICACIÓN DE LA ACTIVIDAD & TIEMPO \\
\hline $\begin{array}{l}\text { Sesión 1. Actividad de } \\
\text { Presentación. }\end{array}$ & $\begin{array}{l}\text { Presentación de la asignatura y de la unidad } 1 \text { (Ciclo } \\
\text { De Mejora Integrado). Dinámica de las clases y } \\
\text { constitución de los equipos de trabajo. }\end{array}$ & $80 \mathrm{~min}$. \\
\hline \multicolumn{3}{|c|}{$\begin{array}{l}\text { SESIÓN 2. Subproblema: ¿Qué características tiene la enseñanza de las CCSS y la } \\
\text { enseñanza Patrimonial? }\end{array}$} \\
\hline TIPO DE ACTIVIDAD & EXPLICACIÓN DE LA ACTIVIDAD & TIEMPO \\
\hline $\begin{array}{l}\text { Cuestionario de ideas } \\
\text { previas }\end{array}$ & Realización del cuestionario de ideas previas & $60 \mathrm{~min}$. \\
\hline $\begin{array}{l}\text { Actividad de Contraste } \\
\text { Ideas previas }\end{array}$ & $\begin{array}{l}\text { ¿Qué Ciencias Sociales y qué Patrimonio aprendiste? } \\
\text { ¿Cómo te lo enseñaron? } \\
\text { Puesta en común en pequeño grupo de las } 3 \\
\text { primeras preguntas del cuestionario inicial y entrega } \\
\text { de un documento de sintesis de ideas del grupo a } \\
\text { partir de las ideas comunes e ideas divergentes. }\end{array}$ & 20 min. \\
\hline \multicolumn{3}{|c|}{ SESIÓN 3. Subproblema: ¿Hay otras formas de enseñar Ciencias Sociales y Patrimonio? } \\
\hline TIPO DE ACTIVIDAD & EXPLICACIÓN DE LA ACTIVIDAD & TIEMPO \\
\hline $\begin{array}{l}\text { Actividad inicial } \\
\text { Perspectivas socio- } \\
\text { patrimoniales y } \\
\text { Modelos didácticos }\end{array}$ & $\begin{array}{l}\text { Introducción de la docente donde se acercará al } \\
\text { alumnado a las distintas perspectivas sociales } \\
\text { sobre las problemáticas que les afectan y diferentes } \\
\text { formas de entender el patrimonio y la enseñanza de } \\
\text { problemas en las escuelas. }\end{array}$ & $5 \mathrm{~min}$. \\
\hline $\begin{array}{l}\text { Actividad de Contraste } \\
1\end{array}$ & $\begin{array}{l}\text { Visionado del vídeo Polonceau } \\
\text { Visionado del vídeo Regreso }\end{array}$ & $15 \mathrm{~min}$. \\
\hline $\begin{array}{l}\text { Actividad de Contraste } \\
2\end{array}$ & $\begin{array}{l}\text { Lectura y análisis del Documento 1- Domínguez } \\
\text { Chillón, G. (1998). Jimmy, ¿por qué estás tan moreno? } \\
\text { Aula de innovación educativa, 75, 1-7. }\end{array}$ & $30 \mathrm{~min}$. \\
\hline
\end{tabular}

Jornadas de Formación e Innovación Docente del Profesorado | № 2 (2019) Esta obra se distribuye con la licencia Creative Commons Reconocimiento-NoComercial-SinObraDerivada Internacional (CC BY-NC-ND 4.0.) 


$$
\begin{array}{|l|l}
\text { Actividad de Contraste } & \begin{array}{l}
\text { Lectura y análisis del Documento 2- Análisis sobre } \\
\text { tres modelos didácticos (tradicional, espontaneísta } \\
\text { y de investigación) donde se trabajan las Ciencias } \\
\text { Sociales con la integración del patrimonio cultural. }
\end{array}
\end{array}
$$

\begin{tabular}{|c|c|c|}
\hline TIPO DE ACTIVIDAD & EXPLICACIÓN DE LA ACTIVIDAD & TIEMPO \\
\hline $\begin{array}{l}\text { Actividad inicial } \\
\text { El modelo didáctico } \\
\text { personal }\end{array}$ & $\begin{array}{l}\text { Se comienza la sesión haciendo un repaso de las } \\
\text { ideas trabajadas a partir de los vídeos y el trabajo de } \\
\text { los tres casos y se dirige el debate hacia la idea de la } \\
\text { definición de los modelos didácticos y de un modelo } \\
\text { didáctico personal. } \\
\text { Primera aproximación al Modelo Didáctico Personal. }\end{array}$ & $10 \mathrm{~min}$. \\
\hline $\begin{array}{l}\text { Actividad de contraste } \\
1\end{array}$ & $\begin{array}{l}\text { Lectura individual del listado de principios } \\
\text { didácticos personales y elección de los principios } \\
\text { afines a un modelo de docencia (Adaptación de la } \\
\text { actividad } 5.1 \text { de Porlán (2017, pp. 94-95). } \\
\text { Definición y justificación de unos principios } \\
\text { didácticos que caractericen un modelo didáctico } \\
\text { personal. }\end{array}$ & $45 \mathrm{~min}$. \\
\hline $\begin{array}{l}\text { Actividad de } \\
\text { conclusión de } \\
\text { subproblema }\end{array}$ & $\begin{array}{l}\text { Puesta en común en gran grupo respondiendo a } \\
\text { la pregunta: ¿Cómo puede un modelo didáctico } \\
\text { personal comprometerse con el cambio y la mejora } \\
\text { educativa? }\end{array}$ & 25 min. \\
\hline \multicolumn{3}{|c|}{$\begin{array}{l}\text { SESIÓN } 5 \text { y SESIÓN 6. Subproblema: ¿Qué entendemos por PSR? ¿Qué relación guardan } \\
\text { loS PSR con la Educación Patrimonial? ¿Y viceversa? ¿Se puede definir un PSR y } \\
\text { Patrimonial para trabajarlo en el aula? }\end{array}$} \\
\hline $\begin{array}{l}\text { Actividad de ideas } \\
\text { previas }\end{array}$ & $\begin{array}{l}\text { Se les pide que, en pequeño grupo, seleccionen una } \\
\text { problemática social/patrimonial. Puede recoger un } \\
\text { problema micro (de su barrio), un problema meso } \\
\text { (de la ciudad o pueblo) y problema macro (del país, } \\
\text { del mundo) o interrelacionado. Una vez tengan esa } \\
\text { selección, deben justificar: } \\
\text { 1. ¿Por qué piensan que eso debería enseñarse en la } \\
\text { escuela? } \\
\text { 2. ¿Qué tipo de aprendizajes, distintos de los } \\
\text { habituales, fomenta? } \\
\text { 3. Por grupo de trabajo, representar gráficamente, } \\
\text { destacando los elementos esenciales y sus } \\
\text { interacciones, que definan qué es un problema } \\
\text { social y/o patrimonial relevante y qué se puede } \\
\text { enseñar a través de él. }\end{array}$ & $\begin{array}{l}160 \\
\min .\end{array}$ \\
\hline
\end{tabular}

SESIÓN 4. Subproblemas: ¿Qué te planteas como maestro/a para poder trabajar un PSPR en el aula? ¿Cómo traspasar mi MDP a mi aula? 
SESIÓN 7. Subproblema: ¿Qué entendemos por PSR? ¿Qué relación guardan loS PSR con la Educación Patrimonial? ¿Y viceversa? ¿Se puede definir un PSR y Patrimonial para trabajarlo en el aula?

\begin{tabular}{|l|l|l|}
\hline TIPO DE ACTIVIDAD & EXPLICACIÓN DE LA ACTIVIDAD & TIEMPO \\
\hline $\begin{array}{l}\text { Actividad de contraste } \\
1\end{array}$ & $\begin{array}{l}\text { Aproximación a los conceptos de Patrimonio } \\
\text { cultural y Problema Social Relevante, realizando } \\
\text { una formulación del concepto Problema Social- } \\
\text { Patrimonial Relevante en el Entorno a partir del } \\
\text { contraste realizado por las profesoras implicadas en } \\
\text { el CIMA con una sesión de trabajo conjunto. }\end{array}$ & 40 min. \\
\hline $\begin{array}{l}\text { Actividad de Contraste } \\
2\end{array}$ & $\begin{array}{l}\text { Lectura y análisis de documentos: } \\
\text { Doc. 3- Instituto Andaluz de patrimonio } \\
\text { histórico. Qué es patrimonio cultural. } \\
\text { Extraído desde http://www.iaph.es/web/canales/ } \\
\text { patrimonio-cultural/ } \\
\text { Doc. 4- Santisteban, A. (2009). Cómo trabajar en } \\
\text { clase la competencia social y ciudadana. Aula de } \\
\text { innovación educativa, 187, 12-15. }\end{array}$ & 40 min. \\
\hline $\begin{array}{l}\text { Actividad de Contraste } \\
3\end{array}$ & $\begin{array}{l}\text { Explicación de las docentes sobre PSPP y resolución } \\
\text { de dudas. }\end{array}$ & 40 min. \\
\hline
\end{tabular}

SESIÓN 8. Subproblema: ¿Qué entendemos por PSR? ¿Qué relación guardan los PSR con la Educación Patrimonial? ¿Y viceversa? ¿Se puede definir un PSR y Patrimonial para trabajarlo en el aula?

\begin{tabular}{|l|l|l|}
\hline $\begin{array}{l}\text { Actividad de } \\
\text { conclusión de } \\
\text { subproblema }\end{array}$ & $\begin{array}{l}\text { Contraste de la representación gráfica realizada en la } \\
\text { sesión anterior con las ideas extraídas de los textos. } \\
\text { Reformulación del gráfico y 3 ideas de conclusión de } \\
\text { qué es un problema sociopatrimonial relevante. }\end{array}$ & 20 min. \\
\hline $\begin{array}{l}\text { Actividad de contraste } \\
1\end{array}$ & $\begin{array}{l}\text { ¿Cómo enseñar mi problema desde una perspectiva } \\
\text { social y patrimonial siguiendo los elementos del } \\
\text { sistema didáctico? } \\
\text { Diapositiva Sistema didáctico y sus interacciones } \\
\text { (Porlán, 2017) }\end{array}$ & 20 min. \\
\hline & $\begin{array}{l}\text { Lectura y análisis de documentos: } \\
\text { Doc. 5- López Martínez, M.J. (2017). Los problemas } \\
\text { relevantes de la sociedad actual en un aula de 5 } \\
\text { años: qué hacemos con las personas refugiadas } \\
\text { sirias. Investigación en la escuela, 92, 19-31. } \\
\text { Doc. 6- Prats, J. y Santacana, J. (2009). Ciudad, } \\
\text { educación y valores patrimoniales. La ciudad } \\
\text { educadora, un espacio para aprender a ser } \\
\text { ciudadanos. Revista Íber, 59, 1-7. }\end{array}$ & 40 min. \\
\hline
\end{tabular}


SESIÓN 9. Subproblema: ¿Qué entendemos por PSR? ¿Qué relación guardan loS PSR con la Educación Patrimonial? ¿Y viceversa? ¿Se puede definir un PSR y Patrimonial para trabajarlo en el aula?

\begin{tabular}{|l|l|l}
\hline Actividad de contraste & Sesión de trabajo conjunta de los estudiantes de & 80 min. \\
& Primaria e Infantil a partir del siguiente guion: & \\
& - Compartir nuestros problemas y proponer mejoras/ & \\
ideas/sugerencias para hacerlo más completo. & - Compartir cómo enseñar nuestro problema en \\
& clase y proponer mejoras/ideas/sugerencias para & \\
& hacerlo más completo. & \\
& - Comentar primeras ideas de cómo realizar el vídeo \\
& que introduzca la problemática. & \\
\hline
\end{tabular}

SESIÓN 10. Problema: ¿Qué problemáticas sociales y patrimoniales hay en tu entorno?

Actividad de

conclusión problema Guion para la realización del vídeo a partir de la $80 \mathrm{~min}$. problemática seleccionada cuya característica fundamental es que va a ser el elemento desencadenante de la problemática en clase, el elemento motivador y de presentación para los niños y niñas.

SESIÓN 11 y 12. Problema: ¿Qué problemáticas sociales y patrimoniales hay en tu entorno?

\begin{tabular}{|l|l|l|}
\hline $\begin{array}{l}\text { Actividad de } \\
\text { conclusión problema }\end{array}$ & \multicolumn{1}{|l|}{$\begin{array}{l}\text { Elaboración de vídeo con sesiones en el aula y en el } \\
\text { exterior para realizar las tomas. }\end{array}$} & $\begin{array}{l}160 \\
\text { min. }\end{array}$ \\
\hline SESIÓN 13. Problema: ¿Qué problemáticas sociales y patrimoniales hay en tu entorno? \\
\hline $\begin{array}{l}\text { Actividad de } \\
\text { conclusión problema }\end{array}$ & $\begin{array}{l}\text { Presentación de las propuestas de cada grupo de } \\
\text { trabajo al resto del grupo clase }\end{array}$ & 80 min. \\
\hline SESIÓN 14. Problema: ¿Qué problemáticas sociales y patrimoniales hay en tu entorno? \\
\hline $\begin{array}{l}\text { Cuestionario de ideas } \\
\text { inales }\end{array}$ & Realización del cuestionario de ideas finales & 40 min. \\
\hline
\end{tabular}

\section{Cuestionario inicial-final de seguimiento de la progresión de las ideas de los estudiantes}

Para poder valorar la progresión en los aprendizajes de los estudiantes, ambas docentes hemos desarrollado una actividad inicial y final que nos ha permitido acercarnos a los modelos e ideas que predominan en los dos grupos de clase. Hemos intentado que las preguntas planteadas 
comenzasen haciendo mención a sus experiencias previas como estudiantes, siguiesen con su posicionamiento como futuros maestros/as en cuestiones relativas al diseño de propuestas didácticas y finalizasen con el esbozo de sus principios didácticos. El instrumento de recogida de ideas ha sido el siguiente:

1. Durante tus estudios en la enseñanza obligatoria ¿has cursado alguna asignatura relacionada con las Ciencias Sociales y el Patrimonio Cultural? Indica cuáles.

2. ¿Qué contenidos estudiaste y qué metodología y recursos empleaban los profesores para ello?

Contenidos de CCSS:

Contenidos de Patrimonio cultural: Metodologías:

3. ¿Existen problemáticas sociales y patrimoniales que pueda ser de interés su enseñanza en la escuela? En caso de respuesta afirmativa, menciona tres y explica por qué las elegiste.

4. Imaginate en un aula de un centro de Educación Infantil y Primaria de Sevilla, en un barrio de la ciudad, quizá en el que naciste o en el pueblo en el que actualmente habitas, piensa que has de enseñar al alumnado el entorno social y patrimonial:

a) define qué entiendes por ambos conceptos

b) enuncia algunos de los objetivos para su enseñanza

c) menciona tres elementos que enseñarías y explica porqué

d) explica qué metodología de enseñanza emplearías

e) cuenta tres actividades que harías para enseñar integradamente las Ciencias Sociales y el patrimonio cultural

f) qué evaluarías de los aprendizajes

5. ¿Qué aprendería un niño o niña con esta propuesta y para qué crees que le serviría? 
6. ¿Qué tipo de maestro/a te gustaría ser? Describe o caracteriza un modelo de enseñanza y unos principios que lo guíen.

\section{Diario de las sesiones}

El inicio del CIMA coincidió con el comienzo del curso académico, por lo que la primera sesión se dedicó a explicar el programa de la asignatura, el funcionamiento, metodología y la vinculación de esta con su futuro perfil profesional. En ese sentido, y dado que tanto los estudiantes como la docente consideramos que conocernos era fundamental para poder trabajar cómodos, dedicamos la mayor parte de esta sesión a presentarnos, hablar un poco de nosotros y a conocer las expectativas que teníamos con respecto a la asignatura. De este modo, la realización del cuestionario de ideas previas no pudo realizarse aquí, y tuvimos que pasarla al inicio de la segunda sesión, lo que implicó un primer retraso en la planificación de la secuencia de actividades. Para los estudiantes del Grado en Educación Primaria la realización de este cuestionario resultó algo pesado, y en numerosas ocasiones hubo que pedir expresamente que trabajasen de forma individual. Además, la pregunta 4 fue extensa y en ocasiones se perdió el sentido que tenía de realizar una propuesta de enseñanza para el entorno social y patrimonial. Esto ha conllevado la modificación de este cuestionario para el próximo curso, propuesta que se detalla más adelante. Este retraso del primer día afectó al resto de la secuencia de actividades, pues hubo que reorganizar e incluso suprimir algunas de las actividades previamente planificadas. En este sentido, y tras las tres primeras sesiones, las docentes vimos conveniente modificar dos sesiones de trabajo. En un principio, la sesión 4 estaba planificada para que los estudiantes, en pequeño grupo, seleccionaran la problemática social y patrimonial que iban a trabajar. Sin embargo, en las sesiones semanales de evaluación que 
las docentes íbamos teniendo para coordinar el trabajo, determinamos que antes de eso, era preciso que reflexionasen sobre los principios que guiarían su práctica docente, que enlazaba mejor con las actividades que hasta el momento se habían estado trabajando (de análisis de modelos didácticos) y permitía poner el foco en un modelo personal de enseñanza. Por tanto, llevamos a cabo el cambio (tal y como puede verse en la tabla 1).

Resultó especialmente complicada la actividad correspondiente a la selección de una problemática social y patrimonial. Para los estudiantes del Grado en Educación Primaria, trabajar problemas siempre se ve desde una connotación negativa, vinculada a elementos que tienen que ver con grandes problemas del mundo (contaminación, cambio climático, hábitos alimenticios, inmigración, etc.), siendo dificil concretar dichos problemas en su entorno más próximo y, sobre todo, vincularlo con cuestiones patrimoniales. En este sentido, esta actividad que estaba planificada para su realización de forma autónoma acabó estando dirigida y revisada por las docentes, ayudándoles a enfocar bien el problema.

En cuanto a aquellas actividades que supusieron el análisis de documentos, funcionaron muy bien pues se habían seleccionado lecturas concretas sobre los problemas que se estaban trabajando y el guion que las acompañaba hizo que se aprovechara el material para el resto de las actividades. Ambas docentes compartimos la impresión que estas actividades han facilitado el aprendizaje por parte de los estudiantes.

De este CIMA integrado se destacan dos actividades por encima del resto: por un lado, que las dos docentes participásemos en una sesión conjunta, trabajando con los estudiantes los contenidos en los que cada una es especialista, que ofreció una visión muy sólida del proceso de coordinación y ayudó a que los estudiantes pudiesen 
reformular los problemas que habían elegido a la luz del contraste que se había hecho; por otro lado, la sesión donde unimos la clase de Infantil con la de Primaria para debatir y mejorar los problemas sociales y patrimoniales. Aunque resultó interesante, pues los trabajos se enriquecieron con la perspectiva aportada desde el otro nivel educativo, conllevó algunos problemas de organización, por el número de estudiantes participantes (110 en total) y la disposición del aula (con mesas ancladas al suelo).

Por último, y en cuanto a la elaboración del vídeo para concluir este primer problema, en su mayoría recogieron buena parte de los contenidos trabajados, sobre todo aquellos vinculados con la definición de problemáticas sociales y patrimoniales cercanos a los niños y niñas de Primaria. Para los estudiantes, esta actividad supuso una buena forma de expresar el aprendizaje alcanzado poniendo en juego otras habilidades -más relacionadas con la creatividad y la expresión artística- y motivándoles para pensar que los contenidos pueden presentarse y organizarse de formas alejadas del libro de texto tradicional.

\section{Evaluación del aprendizaje de los estudiantes}

Para poder evaluar la progresión del aprendizaje de los estudiantes, las docentes utilizamos un cuestionario al inicio y al final que nos ha permitido conocer y analizar los modelos mentales de los estudiantes (véanse figura 5 en adelante). Cabe decir que el resto de los materiales que los estudiantes han producido también forman parte del proceso de evaluación continua de la asignatura, pero cuyos resultados no presentamos aquí por falta de espacio. Como puede verse en la mayoría de las preguntas, los estudiantes han evolucionado en relación con los objetivos de enseñanza planificados, detectándose algunos obstáculos de aprendizaje que es preciso comentar. 
Con respecto al tipo de problemáticas sociales y patrimoniales que la mayoría de los estudiantes seleccionaron en la pregunta 3 en el cuestionario de ideas inicial (Cl1), eran "problemas globales para formar en valores a los alumnos y alumnas" (nivel 2). En el cuestionario final ( $\mathrm{Cl} 2)$, superado el obstáculo de que solo existen problemas a nivel macro, la mayoría se ubicó en el nivel 3, señalando problemáticas vinculadas con la realidad más próxima a los alumnos.

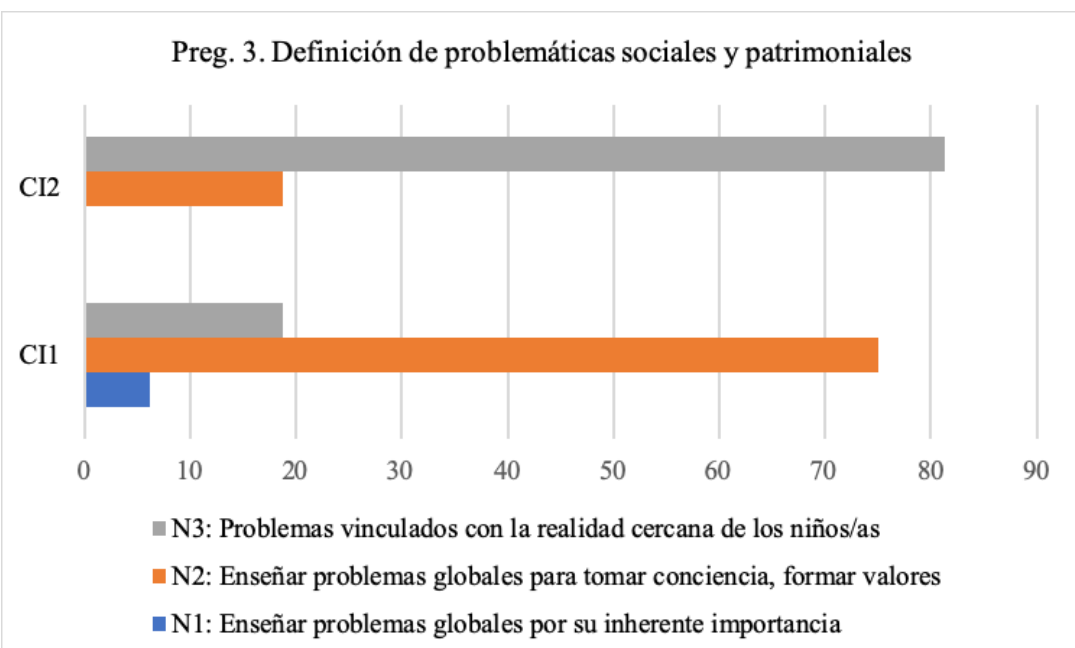

Figura 5. Progresión de los estudiantes en la definición de problemáticas sociales y patrimoniales

Para la pregunta 4a, donde se pedía que caracterizaran el entorno social y patrimonial, la mayoría de los estudiantes han progresado en sus ideas. Destaca de esta pregunta la evolución en el concepto de patrimonio (véase figura 7), donde el cuestionario $\mathrm{Cl} 2$ refleja que la mayoría son capaces de profundizar en los elementos que lo componen, yendo más allá de respuestas genéricas. Esta evolución no se ha dado de la misma forma para el concepto de entorno social (véase figura 6), donde en el $\mathrm{Cl} 2$ aún encontramos un porcentaje alto de estudiantes en los niveles 1 y 2. Detectamos aquí un obstáculo relativo a la compresión del entorno más allá de un espacio físico (un lugar) donde 
ocurre la vida de las personas hacia construir la idea de que es un espacio simbólico de convivencia y también de participación.

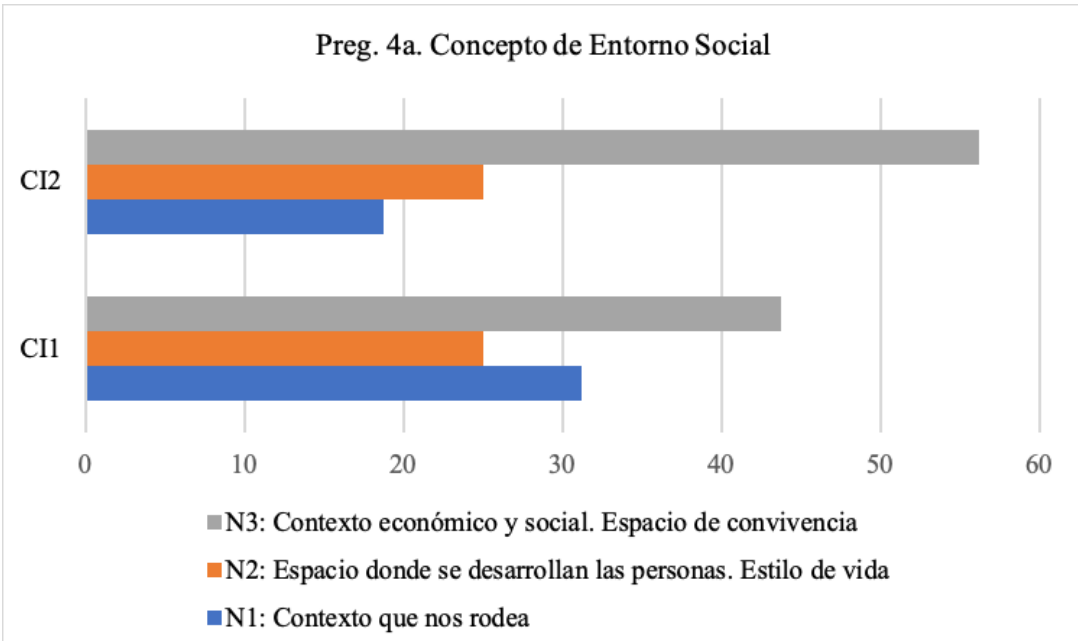

Figura 6. Progresión de los estudiantes en relación con el concepto de Entorno Social

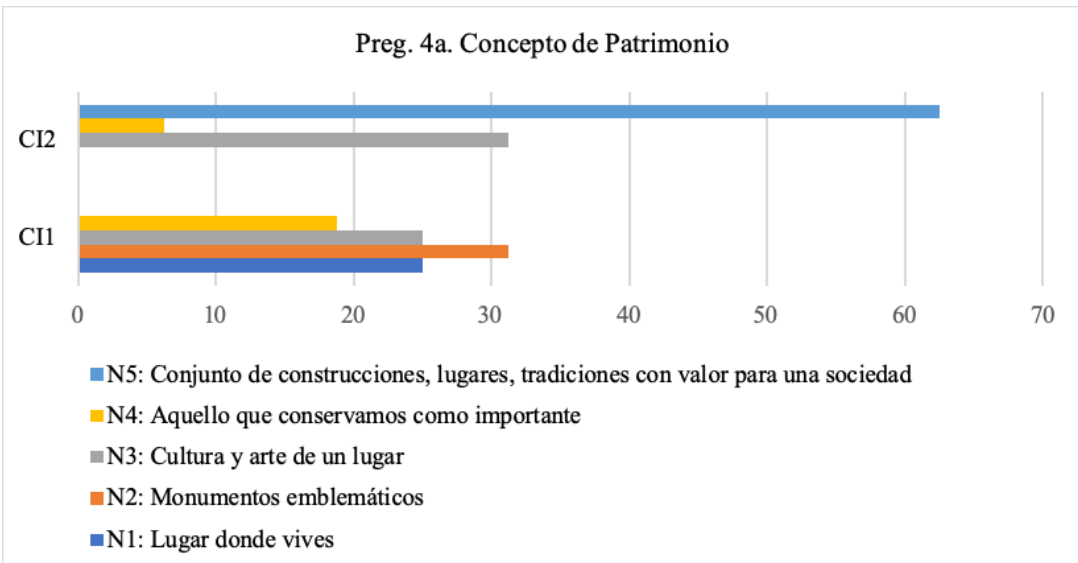

Figura 7. Progresión de los estudiantes en relación con el concepto de Patrimonio

El análisis de la pregunta 4c, relativa a la propuesta de contenidos a enseñar, pone de manifiesto que los estudiantes siguen teniendo dificultades en definir contenidos vinculados con problemas, lo que requiere que en el 
futuro abordemos específicamente esta cuestión. Destaca en esta pregunta que, tras el proceso formativo, los estudiantes consideran que los contenidos que se deben enseñar están relacionados fundamentalmente con monumentos emblemáticos y con las culturas cercanas más estereotipadas, no superando una visión "monumentalista" y "folclórica" del patrimonio y del entorno.

Preg. 4c. Propuesta de contenidos a enseñar

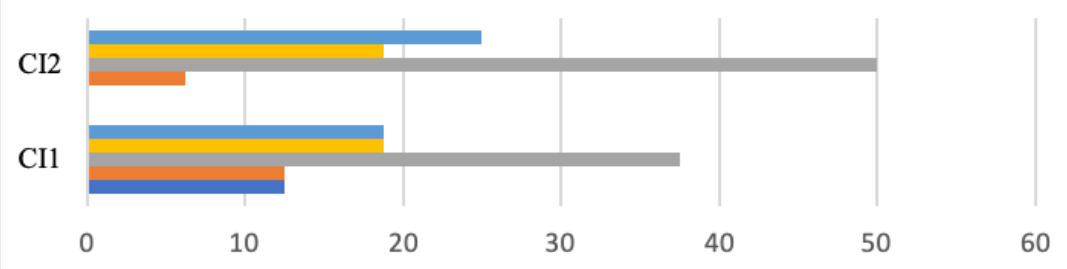

- N4: Contexto cercano: patrimonio arquitectónico, cultural, estilo de vida

N3: Problemas globales (cambio climático, diversidad cultural, etc.)

- N2: Enseñar monumetos y culturas próximas

N1: Contenidos generales

- N0: No contesta

Figura 8. Progresión de los estudiantes en relación con los contenidos a enseñar

Los resultados de la pregunta $4 \mathrm{~d}$, vinculada con la metodología de enseñanza, ponen de manifiesto que el 50\% de los estudiantes es capaz de identificar que para desarrollar una propuesta de actividades con sentido para los alumnos y alumnas de primaria es necesario hacerlo desde el trabajo por problemas relevantes. Sin embargo, es muy destacable, que el otro $50 \%$ de los estudiantes, entiende que una enseñanza alternativa se basa solo en hacer actividades lúdicas e inconexas alejados del libro de texto, pero sin un modelo metodológico coherente y fundamentado. Esta cuestión es necesaria abordarla en el resto de los problemas de la asignatura.

Jornadas de Formación e Innovación Docente del Profesorado | № 2 (2019) Esta obra se distribuye con la licencia Creative Commons Reconocimiento-NoComercial-SinObraDerivada 
Preg. 4d. Metodología de enseñanza

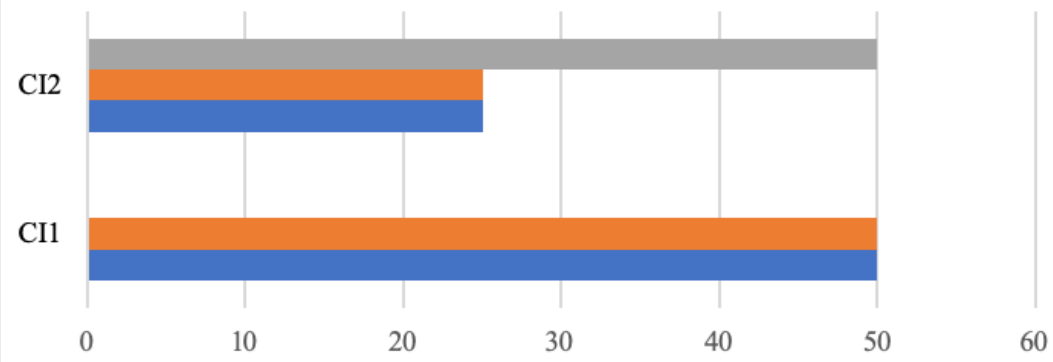

N3: Trabajo por problemas cercanos y relevantes

-N2: Visitas, búsqueda de informacion, proyectos de investigación

-N1: Metodología activa, partiendo de ideas previas

Figura 9. Progresión de los estudiantes en relación con la metodología de enseñanza

Para la pregunta $4 f$, relacionada con la evaluación del proceso de enseñanza y aprendizaje, también detectamos progresión de las ideas de los estudiantes, pasando en el cuestionario Cl1 de un nivel 1, en el que se evalúa solo la adquisición de conocimientos al final, a los niveles 2 y 3 en el $\mathrm{Cl} 2$ en los que se entiende la evaluación como un proceso continuo y basado en relacionar los contenidos trabajados, creando esquemas de aprendizaje.

Preg. 4f. Propuesta de evaluación

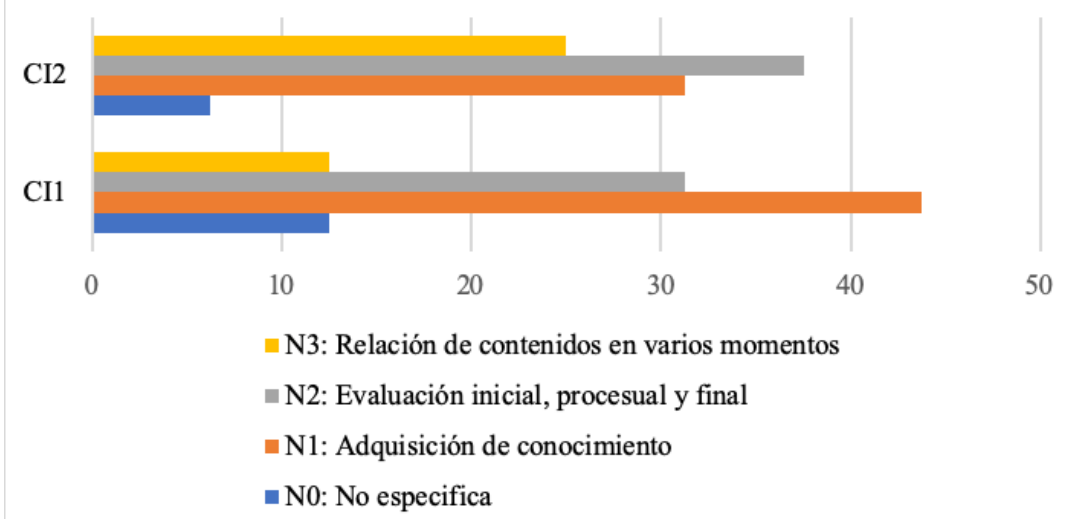

Figura 10. Progresión de los estudiantes en relación con la evaluación

Jornadas de Formación e Innovación Docente del Profesorado | № 2 (2019) Esta obra se distribuye con la licencia Creative Commons Reconocimiento-NoComercial-SinObraDerivada 
En cuanto a las finalidades de la enseñanza, hay un aumento considerable al final hacia el nivel 2, vinculado con enseñar cuestiones relativas al entorno social y patrimonial para desarrollar habilidades para comprender el entorno y formar ciudadanía. Sin embargo, más de un 30\% de los estudiantes aún manifiestan que la finalidad de la enseñanza es solo una cuestión de respeto, haciéndose evidente que no se ha profundizado en la necesidad de entender los procesos de enseñanza y aprendizaje como espacios de participación y formación ciudadana.

Preg. 5. Finalidades de la enseñanza

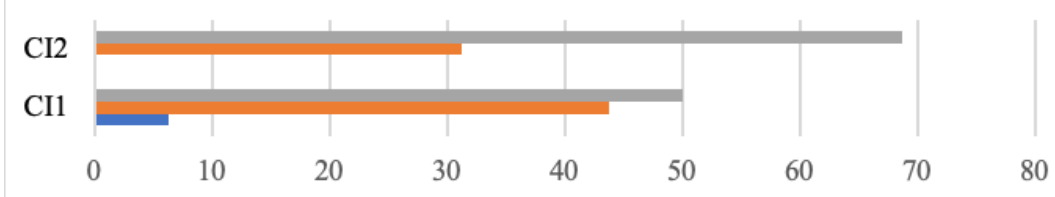

N2: Desarrollo de habilidades para comprender el entorno y formar ciudadanos

N1: Respeto por cultura y monumentos

-N0: No contesta

Figura 11. Progresión de los estudiantes en relación con las finalidades de la enseñanza

Finalmente, para la pregunta 6 donde se les pedía la definición del modelo de enseñanza, los resultados se han mantenido en ambos cuestionarios, poniendo de relieve que la actividad planificada para abordar este problema y contenido ha sido insuficiente y requiere que, para futuros CIMA, se trabaje con más profundidad. 
Preg. 6. Definición de modelo de enseñanza

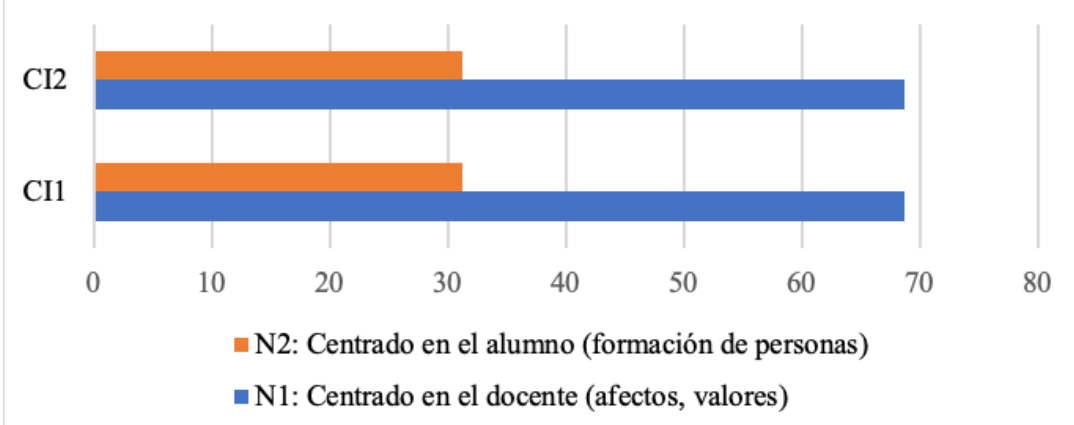

Figura 12. Progresión de los estudiantes en relación con el modelo de enseñanza

\section{Evaluación del CIMA integrado}

La evaluación del CIMA integrado ha sido positiva, sin embargo, a la luz de la progresión de las ideas de los estudiantes, del diario de sesiones de cada una de las profesoras y de las sesiones de evaluación conjunta, determinamos que es necesario mantener e incluir algunas cuestiones para el próximo curso.

Aspectos a mantener:

- La realización del primer problema de la asignatura de forma coordinada nos ha permitido a nosotras como docentes y a los estudiantes pensar que el futuro desarrollo profesional de un maestro, sea de Infantil o de Primaria, requiere de coordinación y consenso en virtud de mejorar la calidad de la enseñanza.

- Consideramos que el mapa de contenidos propuesto y trabajado recoge los elementos esenciales para este primer problema.

- El modelo metodológico presentado se ha desarrollado tal y como estaba previsto y nos ha servido como marco para la elaboración de la secuencia de actividades. 
Las actividades de lectura de documentos, junto con los guiones de trabajo, han resultado útiles para el contraste de ideas.

- Las sesiones de trabajo conjuntas han sido relevantes para el buen desarrollo de las actividades propuestas.

Aspectos a mejorar:

- Es preciso modificar el cuestionario, eliminando las preguntas 1 y 2 , que solo sirven para contextualizar a los estudiantes y que pueden hacerse a partir de otras dinámicas, y reduciendo la pregunta 4 sólo a los apartados a, c, d y f, pues los otros se abordan de forma implícita con otras cuestiones, reduciéndose así el tiempo de la actividad.

- El análisis de la secuencia de actividades en las reuniones de coordinación de las docentes ha llevado a introducir adaptaciones y ajustes sobre la marcha. Aunque la secuencia presentada aquí es la realmente aplicada, a la luz de los resultados de los cuestionarios, consideramos necesario introducir algunas nuevas relacionadas con: la caracterización del entorno social, el análisis de los contenidos a enseñar y la formulación de principios didácticos.

- Para la actividad de selección y justificación de problemáticas sociales y patrimoniales y para organizar mejor la puesta en común entre ambas clases es necesario ofrecer una propuesta única de problemas relacionados.

- La actividad donde las dos docentes estuvimos juntas en el aula debería de pasar de una sesión a tres como mínimo, de forma que podamos hacer un seguimiento conjunto del problema trabajado y una observación más detallada de la evolución de las dos clases.

- La actividad de "compartir experiencias que enseñan", donde unimos las dos clases, es preciso organizarla mejor y buscar un espacio acorde con el tipo de trabajo que se le pedía a los estudiantes. 
- Por último, es necesario ofrecer ejemplos con vídeos introductorios para trabajar los problemas, pues ayudaría a entender el objetivo de la actividad.

\section{Conclusiones}

La realización de un CIMA integrado se ha convertido en una experiencia muy provechosa y enriquecedora. Poder diseñar, dialogar y debatir sobre contenidos y secuencias de actividades, compartir experiencias y evaluar junto con otra compañera el proceso de aprendizaje de los estudiantes y nuestro diseño, ha ofrecido un contexto único a mis estudiantes y a mi. Entender que nuestro trabajo como docentes no puede quedarse sólo en el marco de nuestra clase, sino que debe salir de ella buscando conexiones con otras, implica entender que cambiar la educación universitaria pasa por abrir espacios de reflexión y trabajo conjuntos, para buscar sinergias que provoquen cambios en la institución y en los planes de estudio desde abajo.

Jornadas de Formación e Innovación Docente del Profesorado | № 2 (2019) Esta obra se distribuye con la licencia Creative Commons 


\section{Referencias bibliográficas}

Bolivar, A. (2016). Educar democráticamente para una ciudadanía activa. Revista Internacional de Educación para la Justicia Social (RIEJS), 5(1), 69-87.

García Pérez, F. F. (2000). Un modelo didáctico alternativo para transformar la educación: el modelo de investigación en la escuela. Scripta Nova. Revista Electrónica de Geografia y Ciencias Sociales, (64), 1-26.

Gómez, M., Escofet, A. y Freixa, M. (2014). Los equipos docentes en la educación superior ¿Utopía o realidad? Revista Española de Pedagogía, 258, 509-523.

Navarro-Medina, E. (2017). ¿Qué Ciencias Sociales enseñar en primaria, por qué y para qué? Un ciclo de mejora en el Grado de Educacion Primaria de la Universidad de Sevilla. En R. Porlán y E. Navarro-Medina (Coords.), IV Jornadas de Docencia Universitaria (págs. 599-615). Sevilla: Instituto de Ciencias de la Educacion de la Universidad de Sevilla.

Navarro-Medina, E. (2018). Un ciclo de mejora docente para la formación de maestros y maestras en Didáctica de las Ciencias Sociales. En R. Porlán y E. Navarro-Medina (Coords.), V Jornadas de Docencia Universitaria (págs. 898-921). Sevilla: Instituto de Ciencias de la Educación de la Universidad de Sevilla.

Porlán, R. (2017). Enseñanza universitaria. Cómo mejorarla. Madrid: Editorial Morata.

Jornadas de Formación e Innovación Docente del Profesorado | № 2 (2019) Esta obra se distribuye con la licencia Creative Commons Reconocimiento-NoComercial-SinObraDerivada 4.0 Internacional (CC BY-NC-ND 4.0.) 